\title{
TORSION CLASSES AND A UNIVERSAL CONSTRAINT ON DONALDSON INVARIANTS FOR ODD MANIFOLDS
}

\author{
SELMAN AKBULUT, TOM MROWKA, AND YONGBIN RUAN
}

\begin{abstract}
This paper studies the topology of the gauge group and gives mod 2 universal relations along Donaldson polynomials of smooth 4-manifolds, generalizing Y. Ruan's previous related result.
\end{abstract}

\section{INTRODUCTION AND MAIN THEOREM}

Let $P \rightarrow X^{4}$ be a principal $G$-bundle over a smooth 4-manifold, where $G$ is either $S U(2)$ or $S O(3)$. The space of gauge equivalence classes of irreducible connections on $P, \mathscr{B}^{*}(P)$, is an infinite-dimensional manifold. Given a suitably generic metric on $X$ we can define a finite-dimensional submanifold $\mathscr{M}(P) \subset \mathscr{B}^{*}(P)$ consisting of the equivalences of anti-self-dual (ASD) connections. By a theorem of Freed and Uhlenbeck of the cobordism class of this submanifold is independent of the metric of $X$. In [D2] Donaldson obtained invariants of the smooth manifold $X$ roughly by evaluating the rational cohomology classes of $\mathscr{B}^{*}(P)$ on the fundamental class of $\mathscr{M}(P)$. Hence the understanding of the cohomology structure of $\mathscr{B}^{*}(P)$ plays an important role in this theory. It is known that the rational cohomology of $\mathscr{B}^{*}(P)$ does not give all the cohomology. Even in the case of $X=S^{4}$ the space $\mathscr{B}^{*}(P)$ has a rich structure of torsion cohomology class [AJ]. For the general 4-manifold the torsion classes of $\mathscr{B}^{*}(P)$ have potentially interesting applications, but they are hard to compute [A, R2].

Using the index of a family of Dirac operators, Donaldson was able to construct some 2-torsion homology classes in $\mathscr{B}^{*}(P)$ for $S U(2)$-bundles over spin manifolds. These classes play an important role in the proof of his theorem about intersection forms of spin manifolds, namely:

Theorem [D1]. Let $X$ be a simply connected even 4-manifold. Then if $b_{2}^{+}=1$, the intersection form $q$ is equivalent to $\left(\begin{array}{ll}0 & 1 \\ 1 & 0\end{array}\right)$, and if $b_{2}^{+}(X)=2, q$ is equivalent to $\left(\begin{array}{ll}0 & 1 \\ 1 & 0\end{array}\right) \oplus\left(\begin{array}{ll}0 & 1 \\ 1 & 0\end{array}\right)$.

Another useful application of torsion classes is the torsion invariants defined by Donaldson [D3] (see also [FS]). He used torsion class to define some new invariants of 4-manifolds in order to address the change of smooth structures under connected suming with $S^{2} \times S^{2}$.

Received by the editors February 25, 1993.

1991 Mathematics Subject Classification. Primary 57M99, 57R10; Secondary 58B05, 58D27.

Key words and phrases. Gauge group, Donaldson polynomial, ASD connections, Dirac operator. 
The bulk of this paper is a calculation of $\pi_{1}\left(\mathscr{B}^{*}(P)\right)$ for $S O(3)$, bundles $P \rightarrow X$, generalizing the previous calculations of Freed-Uhlenbeck and Morgan in $S U(2)$ case. This provides us a torsion class in $H_{1}(\mathscr{B} *(P))$. Next we relate these torsion classes to the moduli space $\mathscr{M}(P)$. Taubes's gluing construction gives an imbedding $S O(3) \subset \mathscr{B}^{*}(P)$ which is usually referred to as the "the gluing parameter". The key ingredient in Donaldson's theorem is that his 2-torsion classes detect this gluing parameter. This was accomplished by an excision argument using the families index theorem for elliptic operators. In this paper we take a simple-minded algebraic topological approach and show directly that the generator of $\pi_{1}(S O(3))$ maps nontrivially to the generator of $\pi_{1}\left(\mathscr{B}^{*}(P)\right)$.

As an application of our fundamental group calculation we give a $\bmod 2$ universal constraint for some of Donaldson's invariants of odd manifolds. This generalizes results of the third named author [R1]. Donaldson invariants are infinitely many and are difficult to calculate. Donaldson asked whether there are any universal relations or constraints on Donaldson invariants. Friedman and Morgan showed that the Donaldson invariants of elliptic surfaces and complete intersections are polynomials in their first Chern class and intersection form. They also give a simple relation between Donaldson invariants of complex surface and its blow up.

For each $S O(3)$-bundle $P \rightarrow X$ with first Pontrajagin class $p_{1}$ and second Steifel Whitney class $w_{2}(P)$, it can be shown that Donaldson invariant $\Phi_{w_{2}, p_{1}}\left(\alpha_{1}, \ldots, \alpha_{n}\right)$ is a well-defined integral invariant if $w_{2}\left(\alpha_{i}\right)=0$ for every $\alpha_{i}$. Let us denote the signature of $X$ by $\sigma(X)$. Our main result is

Main Theorem. Let $X^{4}$ be a simply connected manifold with $b^{+}(X) \geq 3$ and odd. Fix $P \rightarrow M$ a principal SO(3)-bundle. If $w_{2}(P)=w_{2}(X)$ and $p_{1}(P)=$ $\sigma(X)+4(\bmod 8)$, then for all $\alpha_{i} \in H^{2}(X)$ with $\alpha_{i} \cdot \alpha_{i} \equiv 0(\bmod 2)$ the $S O(3)$ Donaldson invariants $\boldsymbol{\Phi}_{w_{2}, p_{1}}$ satisfy the constraint:

$$
\begin{aligned}
q \circ \Phi_{w_{2}, p_{1}}\left(\alpha_{1}, \ldots, \alpha_{n}\right) & =\sum_{i<j} q\left(\alpha_{i}, \alpha_{j}\right) \Phi_{w_{2}, p_{1}}\left(\alpha_{1}, \ldots, \widehat{\alpha_{i}}, \ldots, \widehat{\alpha_{j}}, \ldots, \alpha_{n}\right) \\
& \equiv 0(\bmod 2) .
\end{aligned}
$$

Note these hypotheses reduce to that of [R1], when $X$ is spin. For then $w_{2}(T X)=0$ and $\sigma(X) \equiv 0(\bmod 8)$. Thus $P$ lifts to an $S U(2)$-bundle with and $p_{1}=-4 k-4 \equiv 0(\bmod 8)$; then $k$ is odd. As a corollary we also have that in certain cases the zero-degree Donaldson invariants must be even:

Corollary. Under the same assumptions as in the main theorem, if also $b_{2}(X) \geq$ 3 , the zero-degree Donaldson invariant satisfies the condition $\Phi_{0}=0 \bmod 2$.

These constraints severely restrict the value of $\bmod 2$ Donaldson invariants. Fintushel and Stern [FS] have completely calculated the parity of $S U(2)-$ Donaldson invariants for certain cases by using [R1]. Presumably a similar theorem is true for the $S O(3)$-Donaldson invariants based on our results, but we shall not pursue this line here. Our proof of the main theorem follows by applying our calculation to the cobordism construction of [R1]. The torsion class provided by $\pi_{1}\left(\mathscr{B}^{*}\right)$ is crucial in the construction of Donaldsons' torsion invariants. It is straightforward to extend the torsion invariants for odd manifolds. 
The main point of this paper is to prove these results by using purely algebraic topology methods (the first four sections). In $\S 5$ we also give a parallel treatment of this results by using the index theory. But we would like to emphasize that the index theory methods cannot be generalized to other torsion classes whereas the algebraic topological methods do [A].

\section{TOPOLOGY OF $\mathscr{B}^{*}(P)$}

In this section we determine $\pi_{1}\left(\mathscr{B}^{*}(P)\right)$ using obstruction theory. We follow the notes of [M] closely. Let $X$ be a closed smooth 4-manifold with $\pi_{1}(X)=0$ and $P \rightarrow X$ be a principal $G$-bundle, where $G=S O(3)$ or $S U(2)$. We adopt the convention that $G$ acts freely on $P$ by the right multiplication. Let $\mathscr{A}(P)$ be the space of connections on $P$, and let $\mathscr{A}^{*}(P)$ denote the subspace of the irreducible connections on $P$. Let $\mathscr{G}(P)$ be the gauge group, i.e.,

$$
\begin{aligned}
& \mathscr{G}(P)=\{h: P \rightarrow P \mid h(p g)=h(p) g\}, \\
& \mathscr{G}(P)=\left\{s: P \rightarrow G \mid s(p g)=g^{-1} s(p) g\right\} .
\end{aligned}
$$

To be more precise we complete these spaces in appropriate Sobolev norms. The gauge group acts on $\mathscr{A}(P)$ in the obvious way: we denote this action by $(A, h) \rightarrow h^{*} A$. The group $\hat{\mathscr{G}}(P)=\mathscr{G}(P) /\{ \pm I\}$ when $G=S U(2) \quad(\hat{\mathscr{G}}(P)=$ $\mathscr{G}(P)$ when $\left.G=S O_{3}\right)$ acts on $\mathscr{A}^{*}(P)$ freely. Also the reduced gauge group $\mathscr{G}_{0}(P)=\left\{h|h| P_{x_{0}}=\right.$ Id $\}$ acts on $\mathscr{A}(P)$ freely, where $P_{x_{0}}$ denotes the fiber over a base point $x_{0}$. So we get the principal fibrations:

$$
\begin{aligned}
\hat{\mathscr{G}}(P) \rightarrow \mathscr{A}^{*}(P) \rightarrow \mathscr{A}^{*}(P) / \hat{\mathscr{G}}(P) & =\mathscr{B}^{*}(P), \\
\mathscr{G}_{0}(P) \rightarrow \mathscr{A}(P) \rightarrow \mathscr{A}(P) / \mathscr{G}_{0}(P) & =\mathscr{B}_{0}(P) .
\end{aligned}
$$

For simplicity the dependence of these spaces on $P$ often will be dropped from their notation. Since $\mathscr{A}(P)$ and $\mathscr{A}^{*}(P)$ are contractible, we can denote the base spaces as the classifying spaces of the corresponding gauge groups: $\mathscr{B}^{*}(P)=B_{\widehat{G}}$ and $\mathscr{B}_{0}(P)=B_{\mathscr{G}_{0}}$. We can also identify $\mathscr{B}_{0}$ with $\mathscr{A} \times \mathscr{G} P_{x_{0}}$, i.e., it is the equivalence classes of points in the product under the equivalence relation $(A, p) \sim\left(s^{*} A, p . s(p)\right)$ for $s \in \mathscr{G}$.

By considering the restriction map from $\mathscr{G}$ to the automorphisms of a single fiber of $P$ we get the short exact sequence

$$
I \rightarrow \mathscr{G}_{0} \rightarrow \mathscr{G} \rightarrow \operatorname{Aut}\left(P_{x_{0}}\right)=S U(2) \rightarrow I .
$$

Since $S U(2) /\{ \pm I\}=S O(3)$, we get the exact sequence

$$
I \rightarrow \mathscr{G}_{0} \rightarrow \hat{\mathscr{G}} \rightarrow S O(3) \rightarrow I .
$$

So, in particular, $\hat{\mathscr{G}} / \mathscr{F}_{0}=S O(3)$. This induces the exact sequence of fibrations

$$
\mathscr{G}_{0} \rightarrow \widehat{\mathscr{G}} \rightarrow S O(3) \rightarrow B_{\mathscr{S}_{0}} \rightarrow B_{\widehat{\mathscr{G}}} \rightarrow B_{S O(3)} .
$$

Proposition 1. Let $P \rightarrow X$ be a principal G-bundle of a compact smooth 4manifold $X$, with $S O(3)$. Then there is a weak homotopy equivalence:

$$
\mathscr{B}^{*}(P) \simeq \operatorname{Map}^{P}\left(X ; B_{S O(3)}\right)
$$


where the right-hand side denotes the component of the space of (unbased) maps inducing the bundle $P$. If $G=S U(2)$, then $\mathscr{B}^{*}(P)$ is the total space of a covering space with fiber $H^{1}\left(X ; Z_{2}\right)$ :

$$
H^{1}\left(X ; Z_{2}\right) \rightarrow \mathscr{B}^{*}(P) \rightarrow \operatorname{Map}^{P}\left(X ; B_{S O(3)}\right) .
$$

Proof. Let $G=S O(3)$; then consider the principal $G$-bundle:

$$
\xi_{P}=\mathscr{A}^{*} \times{ }_{\hat{\mathscr{G}}} P \rightarrow \mathscr{B}^{*} \times X .
$$

The total space is the equivalence classes of points $[A, p]$ in $\mathscr{A}^{*} \times P$ identified by the equivalence relation $(A, p) \sim\left(s^{*} A, p . s(p)\right)$ where $s \in \hat{\mathscr{G}}$. The $S O(3)$ action on the total space is given by the right multiplication; i.e., if $g \in S O(3)$ and $[A, p] \in \mathscr{A}^{*} \times \widehat{\mathscr{g}} P$, then $[A, p] g=[A, p g]$. The action is well defined since $[A, p] g=[A, p g]=\left[s^{*} A, p g \cdot s^{*}(p g)\right]=\left[s^{*} A, p g \cdot g^{-1} s(p) g\right]=$ $\left[s^{*} A, p . s(p) g\right]=\left[s^{*} A, p . s(p)\right] g$. It is free, since if $[A, p]=[A, p g]$, then $(A, p . g)=\left(s^{*} A, p . s(p)\right)$ for some $s$, so $s=I$ since $A$ is irreducible; so $p g=p$ and therefore $g=I$.

Now consider the classifying maps to the universal $S O(3)$-bundle:

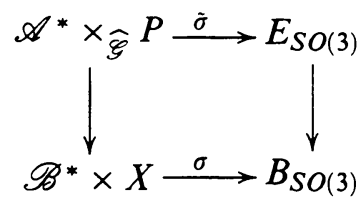

where $\tilde{\sigma}$ is an $S O(3)$-equivariant map and the diagram commutes. This in turn induces the following commutative diagram:

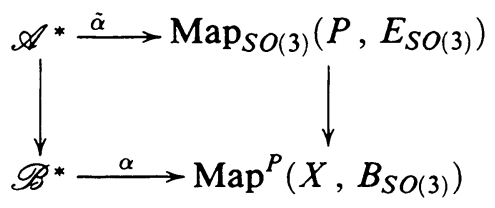

where $\operatorname{Map}_{S O(3)}\left(P, E_{S O(3)}\right)$ denotes the set of $S O(3)$-equivariant maps, and

$$
\tilde{\alpha}(A)(p)=\tilde{\sigma}[A, p] \quad \text { and } \quad \alpha([A])(x)=\sigma([A], x) .
$$

Since $\tilde{\alpha}(A)(p g)=\tilde{\sigma}[A, p g]=\tilde{\sigma}([A, p] g)=(\tilde{\sigma}[A, p]) g=(\tilde{\alpha}(A)(p)) g, \quad \tilde{\alpha}$ is well defined. Furthermore, each vertical map of the diagram principal $\hat{\mathscr{G}}$-bundle map, where the action of $\mathscr{G}$ on $\operatorname{Map}_{S O(3)}\left(P, E_{S O(3)}\right)$ is defined by $(s, f) \mapsto s^{*} f$ with $\left(s^{*} f\right)(p)=f\left(p . s^{-1}(p)\right)$; and $\tilde{\alpha}$ is $\hat{\mathscr{G}}$ equivariant since

$$
\begin{aligned}
\tilde{\alpha}\left(s^{*} A\right)(p) & =\tilde{\sigma}\left[s^{*} A, p\right]=\tilde{\sigma}\left[\left(s^{-1}\right)^{*} s^{*} A, p \cdot s^{-1}(p)\right] \\
& =\tilde{\sigma}\left[A, p \cdot s^{-1}(p)\right]=\tilde{\alpha}(A)\left(p . s^{-1}(p)\right)=s^{*}(\tilde{\alpha}(A))(p) .
\end{aligned}
$$

Since $E_{S O(3)}$ is contractible, so is $\operatorname{Map}_{S O(3)}\left(P, E_{S O(3)}\right)$ and hence both of these are universal principal $\hat{\mathscr{G}}$-bundles. Therefore, the base spaces are weakly homotopy equivalent.

In case $G=S U(2)$ we let $\rho: P \rightarrow \bar{P}$ denote the reduction map to the underlying $S O(3)$-bundle; then we have the basic exact sequence [M]

$$
Z_{2} \rightarrow \mathscr{G}(P) \rightarrow \mathscr{G}(\bar{P}) \stackrel{\alpha}{\longrightarrow} H^{1}\left(X ; Z_{2}\right) \rightarrow 1
$$


where $\alpha$ is defined as follows: for any element $h: \bar{P} \rightarrow \bar{P}$ of $\mathscr{G}(\bar{P})$ we let $\alpha(h)$ be the difference of the two spin structures $\rho$ and $h \circ \rho$ on $\bar{P}$.

Now the proof proceeds just as before. Since the center $\{ \pm I\}$ of $\mathscr{G}(P)$ acts trivially on $\mathscr{A}^{*}$, we get an $S O(3)$-bundle as in $(*)$. The only exception is that in the diagram $(* *)$ the left vertical map is a principal $\hat{\mathscr{G}}(P)$-bundle and the right vertical map is a principle $\hat{\mathscr{G}}(\bar{P})$-bundle. Hence to get a principal $\hat{\mathscr{G}}(P)$-bundle on the right side we must divide $\operatorname{Map}_{S O(3)}\left(P, E_{S O(3)}\right)$ only by the subgroup $\mathscr{G}(P)$ of $\mathscr{G}(\bar{P})$. This gives us $\mathscr{B}^{*}(P)$. Since $\operatorname{Map}^{P}\left(X, B_{S O(3)}\right)$ is obtained from $\operatorname{Map}_{S O(3)}\left(P, E_{S O(3)}\right)$ by dividing $\mathscr{G}(\bar{P}), \operatorname{Map}^{P}\left(X, B_{S O(3)}\right)$ is obtained from $\mathscr{B}^{*}(P)$ by dividing $\mathscr{G}(\bar{P}) / \mathscr{G}(P)$, which is $H^{1}\left(X ; Z_{2}\right)$.

We can now interpret the homotopy groups of $\mathscr{B}^{*}(P)$ in a geometric way:

$$
\begin{aligned}
\pi_{k}\left(\mathscr{B}^{*}\right) & =\left[S^{k}, s_{0} ; \operatorname{Map}^{P}\left(X, B_{S O(3)}\right), P\right] \\
& =\pi_{0} \operatorname{Map}_{*}\left(S^{k}, \operatorname{Map}^{P}\left(X, B_{S O(3)}\right)\right) \\
& =\pi_{0} \operatorname{Map}\left(S^{k} \times X, B_{S O(3)}\right)^{P} \\
& =\left[S^{k} \times X, B_{S O(3)}\right]^{P}
\end{aligned}
$$

where $s_{0}$ denotes the base point of $S^{k}$ and the exponatial $P$ means the set of maps that restrict to $P$ on $s_{0} \times X$ and homotopic to $P$ on each slice $s \times X$ (by viewing view $P$ as a map). Thus we are reduced to the study of isomorphism classes of bundles over $S^{k} \times X$ which restrict to a bundle isomorphic to $P$ on each slice and equal to $P$ on a particular slice:

$$
\pi_{k}\left(\mathscr{B}^{*}\right)=\left\{\downarrow_{S^{k} \times X}^{\xi}|\xi|_{s_{0} \times X}=P,\left.\xi\right|_{s \times X} \cong P \text { for each } s \in S^{k}\right\} .
$$

Theorem 1. Let $P \rightarrow X$ be a principle $S O(3)$-bundle over a compact smooth 4-manifold $X$, with $H_{1}(X)=0$; then

$$
\pi_{1}\left(\mathscr{B}^{*}(P)\right)=\left\{\begin{array}{ll}
Z_{2} & \text { if }\left\{\begin{array}{c}
p_{1}(P)=\sigma(X) \bmod 8 \\
w_{2}(P)=w_{2}(T X) \bmod 2
\end{array}\right\} \\
0 \quad \text { otherwise. }
\end{array}\right\}
$$

Remark. Notice that when $G=S U(2)$ we have $w_{2}(P)=0 \bmod 2$. Hence the theorem in this case reduces to the well-known result:

$$
\pi_{1}\left(\mathscr{B}^{*}(P)\right)= \begin{cases}Z_{2} & \text { if } c_{1}(P)=0 \bmod 2, \text { and } X \text { is even } \\ 0 & \text { otherwise. }\end{cases}
$$

Definition. When $\pi_{1}\left(\mathscr{B}^{*}\right)=Z_{2}$ we denote the corresponding generator of $H^{1}\left(\mathscr{B}^{*} ; Z_{2}\right)=Z_{2}$ by $u_{1}$.

Proof. By the above description, the fundamental group is identified by the isomorphism class of $S O(3)$-bundles over $S^{1} \times X$ which restrict to $P$ on $s_{0} \times X$ and isomorphic to $P$ on all other slices. To count these bundles we cut $S^{1} \times X$ open along $s_{0} \times X$ and count the bundles on $I \times X$ which restrict to $P$ on the boundary. But since $H^{4}\left(X \times S^{1}\right) \approx H_{1}(X) \oplus Z$ has no 2-torsion, by [DW] the 


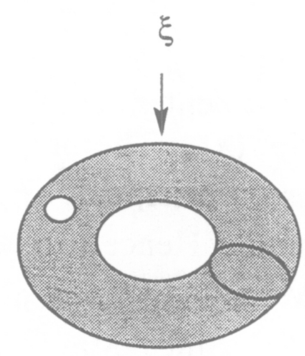

$S^{1} \times S^{1} \times X-\operatorname{int}\left(B^{6}\right)$

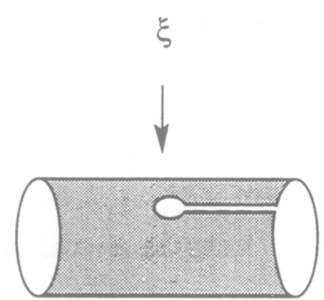

$I \times S^{1} \times X$

FIGURE 1

restriction of these bundles over the 4-skeleton $X \times S^{1}-B^{5}$ are determined by their first Pontryagin and the second Steifel-Whitney classes. But since $H_{1}(X)=$ 0 , the only possibility for these classes are $p_{1}(P) \times 1$ and $w_{2}(P) \times 1$. Therefore the restrictions of these bundles to $X \times S^{1}-B^{5}$ are unique. Furthermore, there are two different ways of extending any bundle over $X \times S^{1}-B^{5}$ to the top skeleton since $\pi_{4} S O(3)=Z_{2}$. Hence if $\eta \rightarrow S^{5}$ is the nontrivial bundle given by the generator of $\pi_{4} S O(3)=Z_{2}$, then

$$
\pi_{1}\left(\mathscr{B}^{*}\right)= \begin{cases}0 & \text { if } P \# \eta \cong P, \\ Z_{2} & \text { otherwise }\end{cases}
$$

where $P \rightarrow S^{1} \times X$ also denotes the bundle pulled back from $P \rightarrow X$ by the projection map and $P \# \eta$ is obtained from $P$ by clutching $P$ with $\eta$ in a chart. The following is an observation of J. Morgan [M]:

Lemma 1. If there is an $S O(3)$-bundle $\xi \rightarrow S^{1} \times S^{1} \times X-\operatorname{int}\left(B^{6}\right)$, such that the restriction to the boundary $\left.\xi\right|_{S^{s}}=\eta$ and $\left.\xi\right|_{0 \times S^{1} \times X}=P$, then $\pi_{1}\left(\mathscr{B}^{*}\right)=0$ otherwise $\pi_{1}\left(\mathscr{B}^{*}\right)=Z_{2}$.

Proof. Given such a bundle $\xi$ we cut $S^{1} \times S^{1} \times X-\operatorname{int}\left(B^{6}\right)$ open to get a bundle of $I \times S^{1} \times X$ which restricts to $P$ on the two outside boundary components and to $\eta$ over $S^{5}$. Then by connecting summing $S^{5}$ to one of the outside boundary components we get a bundle over $I \times X$ restricting $P$ and $P \sharp \eta$ over the two ends; hence, these two bundles are isomorphic. The converse of this also holds. The result follows from the above description of $\pi_{1}\left(\mathscr{B}^{*}\right)$. See Figure 1 .

Now the proof of Theorem 1 follows from the following lemma:

Lemma 2. Every bundle $\xi \rightarrow S^{1} \times S^{1} \times X-\operatorname{int}\left(B^{6}\right)$ with $\xi_{0 \times S^{1} \times X}=P$ extends over $S^{1} \times S^{1} \times X$ if and only if $p_{1}(P)=c_{1}^{2}(P) \bmod 8$, and $c_{1}(P)=$ $c_{1}(T X) \bmod 2$, where $c_{1}(P)$ is an integral lifting of $w_{2}(P)$.

Proof. Call $\left(Z, Z_{0}\right)=\left(S^{1} \times S^{1} \times X, S^{1} \times S^{1} \times X-\operatorname{int}\left(B^{6}\right)\right)$. Since $w_{2}$ has an integral lifting, $\xi$ comes from a $U(2)$-bundle. So we can assume that $\xi$ is a $U(2)$ bundle. In particular, $c_{2}(P)=\left(c_{1}^{2}(P)-p_{1}(P)\right) / 4$. The obstruction to extending the bundle $\xi \rightarrow Z_{0}$ over the whole six-skeleton $Z$ is given by $k_{6}$ where $k_{6}$ is the first $k$-invariant of $B U(2)$. By the definition, $k_{6} \in H^{6}\left(K(Z, 2) \times K(Z, 4), Z_{2}\right)$ such that its pull-back to $B U(2)$ vanishes. $H^{6}\left(K(Z, 2) \times K(Z, 4), Z_{2}\right)$ is generated by $c_{1}^{3}, S q^{2} c_{2}, c_{1} c_{2}$. It is easy to check that

$$
k_{6}=S q^{2} c_{2}(\xi)+c_{1}(\xi) c_{2}(\xi)
$$


is the only choice. We can write

$$
\begin{aligned}
& c_{2}(\xi)=1 \times 1 \times c_{2}(P)+\left[S^{1}\right] \times\left[S^{1}\right] \times \alpha, \\
& c_{1}(\xi)=1 \times 1 \times c_{1}(P)+\varepsilon\left(\left[S^{1}\right] \times\left[S^{1}\right] \times 1\right)
\end{aligned}
$$

where $\alpha \in H^{2}(X)$ and $\varepsilon=0$ or 1 , and obtain

$$
k_{6}=\left[S^{1}\right] \times\left[S^{1}\right] \times\left(\alpha^{2}+\alpha c_{1}(P)+\varepsilon c_{2}(P)\right) .
$$

Hence $k_{6}$ is zero for all such bundles only if for all $\alpha \in H^{2}(X)$

$$
\alpha^{2}+\alpha c_{1}(P)=0 \bmod 2 \text { and } c_{2}(P)=0 \bmod 2 .
$$

By Wu's formula the first condition is equivalent to $c_{1}(P)=c_{1}(T X) \bmod 2$, and the second condition is obviously equivalent to $p_{1}(P)=c_{1}^{2}(P) \bmod 8$.

It remains to show that vanishing $k_{6}$ is sufficient for extending $\xi$ to $Z$. Since if $k_{6}=0$, the obstruction theory says that $\xi$ can be extended only after possibly changing it over the 5-cells, let us show that this is not necessary. That is, if $\xi$ does not extend, then it cannot be extended even after readjusting it over the 5-cells. Otherwise we would have two bundles over the complement of the four-skeleton:

$$
Z_{0}-T^{2} \times X^{(2)} \cup \operatorname{int}\left(B^{2}\right) \times Y=T_{0}^{2} \times Y-\operatorname{int}\left(B^{6}\right)
$$

agreeing over the outside boundary, and one extending the other not extending over $B^{6}$. Here $X^{(2)}$ is the two-skeleton of $X, Y=X-X^{(2)}, T^{2}$ is the 2-torus, $B^{2}$ is a ball in $T^{2}$, and $T_{0}=T-\operatorname{int}\left(B^{2}\right)$. By putting these bundles together on the double of this manifold (two copies of the manifold glued along the common boundary) we obtain a bundle on

$$
W=\operatorname{Double}\left(T_{0}^{2} \times Y\right)-\operatorname{int}\left(B^{6}\right)
$$

which is nontrivial on the boundary. Since $H_{1}(X)=0, Y$ is a homology 4-ball, and hence $W$ is a homology $S^{1} \times S^{5} \# S^{1} \times S^{5}$ minus a point. Hence we get a map $f: W \rightarrow B_{S O(3)}$ which restricts to the nontrivial element of $\pi_{5}\left(B_{S O(3)}\right)=Z_{2}$ on the boundary $S^{5}$. By surgering $\pi_{1}(W)$ we can turn $f$ into a map from a homology ball $f: B^{6} \rightarrow B_{S O(3)}$, contradicting the fact that $f$ is essential on the boundary $S^{5}$. See Figures 2 and 3.

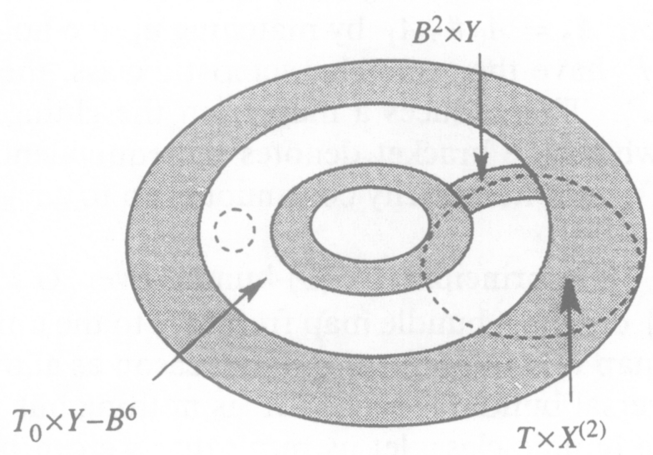

FIGURE 2 


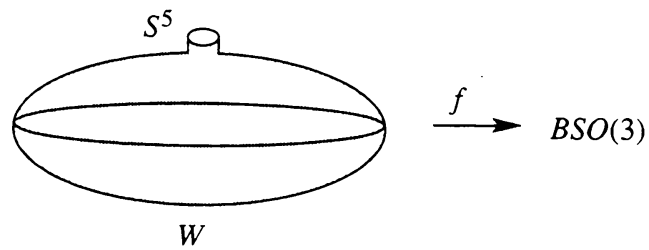

Figure 3

\section{GLUing PARAMETer}

Let $P \rightarrow X$ be an $S O(3)$-bundle over a smooth Riemannian manifold $(X, g)$. Recall that the moduli space $\mathscr{M}$ of ASD connections on $P$ is the space:

$$
\mathscr{M}(P)=\left\{[A] \in \mathscr{B}^{*}(P) \mid F_{+}(A)=0\right\}
$$

where $F_{+}(A)$ is the self-dual part of the curvature of the connection $A$ with respect to metric $g$. Since $S O$ (3)-bundles over 4-manifolds are classified by their first Pontryagin and the second Steifel-Whitney classes, $\mathscr{M}(P)$ is sometimes also denoted by $\mathscr{M}_{w_{2}, p_{1}}$.

The gluing parameter plays a crucial role in the compactification of the moduli space. Taubes's gluing construction gives an embedding

$$
G \rightarrow \mathscr{M} \subset \mathscr{B}^{*} .
$$

The question addressed in this section is how much does $G$ detect the algebraic topology of $\mathscr{B}^{*}$. By the previous section, understanding the homotopy class of this embedding is equivalent to studying the pull-back of the universal bundle over $\mathscr{B}^{*} \times X$. We shall see that this bundle is just a by-product of the gluing construction.

Next, we give a description of Taubes's gluing construction by Donaldson which is more suitable for our setting. Suppose that $X$ is expressed as a union of open sets $X=Z_{0} \cup Z_{1}$ with $\pi_{1}\left(Z_{0} \cap Z_{1}\right)=1$ and $A_{0}$ and $A_{1}$ are $S O(3)$ connections on bundles $P_{0}, P_{1}$ over $Z_{0}, Z_{1}$, respectively, which are both flat on the overlap $Z_{0} \cap Z_{1}$. It $z$ is a base point in $Z_{0} \cup Z_{1}$, then a $S O(3)$ isomorphism

$$
\rho:\left(P_{0}\right)_{z} \rightarrow\left(P_{1}\right)_{z}
$$

defines, by parallel transport, an isomorphism $P_{0}\left|z_{0} \cap z_{1} \cong P_{1}\right| z_{0} \cap z_{1}$. Using this gluing map we construct a bundle $P_{\rho}=P_{0} \cup_{\rho} P_{1}$ over $X$. There is an obvious way to define a connection $A_{\rho}=A_{0} \#_{\rho} A_{1}$ by matching up the holonomy on the overlap. Since $P_{\rho}$ and $P$ have the same characteristic class, there are bundle isomorphism $I_{\rho}: P_{\rho} \rightarrow P$. This induces a map from the gluing parameter to $\mathscr{B}^{*}$ by $\rho \rightarrow\left[I_{\rho}\left(A_{\rho}\right)\right]$, where the bracket denotes the equivalence of the connection. Even though $I_{\rho}$ is not necessarily continuous, up to gauge equivalence $I_{\rho}\left(A_{\rho}\right)$ is smooth.

Let $P_{G}=\bigcup_{\rho} P_{\rho} . \quad P_{G}$ is a principal $S O(3)$-bundle over $G \times X$. For any $v_{\rho} \in P_{\rho},\left[I_{\rho}\left(A_{\rho}\right), I_{\rho}\left(v_{\rho}\right)\right]$ defines a bundle map from $P_{G}$ to the universal bundle $\xi_{P}$ over $\mathscr{B}^{*} \times X$. This map is smooth for the same reason as above. Therefore the pull-back of the universal bundle over $G \times X$ is nothing but $P_{G}$.

In order to classify the bundle class, let us recall that we can build a bundle skeleton by skeleton. Since $X$ is simply connected, we can assume that $X$ 


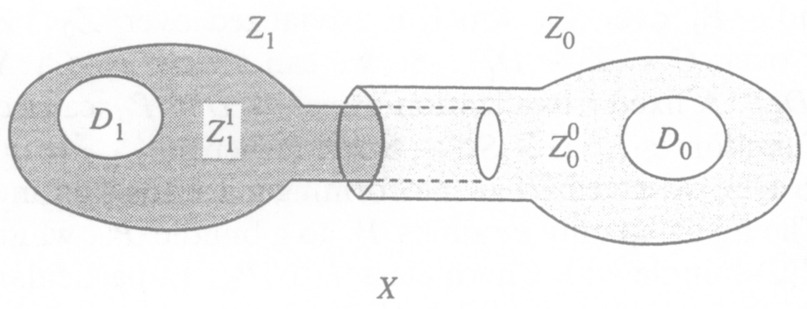

Figure 4

only has 0 -, 2- and 4-cells. In particular, $X-D^{4}$ is the deformation retract of its 2-skeleton. So we can build a bundle in following way: we first build a bundle over the 2-skeleton and then extend over $X-D^{4}$. The obstruction to extending this bundle over $D^{4}$ is the transition function from $X-D^{4}$ to $D^{4}$. This transition function is a map from $S^{3}$ to $S O(3)$ which is classified by its degree. Let $\sigma_{k}$ denote the map of degree $k$. Now we are in the position to give $P_{G}$ a standard description. Let us first consider the case of $w_{2}=0$. The general case will be just a minor modification of this special case. In this case a $S O$ (3)-bundle lifts to a $S U(2)$-bundle and hence is trivial in the complement of a ball. The bundle is completely classified by transition function $\sigma_{k}$. Therefore, topologically we can find trivializations over the punctured 4-manifold $Z_{0}^{0}$ and a 4-ball $D_{0}^{4}$, i.e.,

$$
\left.P_{0}\right|_{Z_{0}^{0}} \cong Z_{0}^{0} \times S O(3),\left.\quad P_{0}\right|_{D_{0}^{4}} \cong D_{0}^{4} \times S O(3) .
$$

The transition function from $Z_{0}^{0}$ to $D_{0}^{4} g_{0,0}: S_{0}^{3} \rightarrow S O(3)$ is $\sigma_{k}$ where $k=$ $c_{2}\left(A_{0}\right)$. In the same way, $Z_{1}=Z_{1}^{1} \cup D_{1}^{4}$ such that

$$
\left.P_{1}\right|_{Z_{1}^{1}} \cong Z_{1}^{1} \times S O(3),\left.\quad P_{1}\right|_{D_{1}^{4}} \cong D_{1}^{4} \times S O(3),
$$

and the transition function $g_{1,1}=\sigma_{l}$ where $l=c_{2}\left(A_{1}\right)$. Clearly from the construction of $P_{G}$ we can conveniently cover $G \times X$ by $G \times D_{0}^{4}, G \times Z_{0}^{0}, G \times$ $Z_{1}^{1}, G \times D_{1}^{4}$. By choosing the obvious trivializations over those open sets, the transition functions become

$$
\begin{aligned}
& g_{0,0}=1 \times \sigma_{k}: G \times S_{0}^{3} \rightarrow S O(3), \\
& g_{0,1}=\rho \times 1: G \times\left(Z_{0} \cap Z_{1}\right) \rightarrow S O(3), \\
& g_{1,1}=1 \times \sigma_{l}: G \times S_{1}^{3} \rightarrow S O(3),
\end{aligned}
$$

where $\rho$ is the gluing parameter. See Figure 4 .

Now we can choose a different local trivialization of the same bundle over $G \times Z_{1}^{1}$ by $\rho^{-1} \times 1$. Then we get a new transition function $g_{0,1}=$ Id and $g_{1,1}=\sigma_{l} \rho$. Because $g_{0,1}=\mathrm{Id}$, we can view $Z_{0}^{0} \cup Z_{1}^{1}$ as a single open set, and bundle restricted to it has a single local trivialization. We can perform the same process for $g_{0,0}$ and note that $S_{0}^{3}$ has a different orientation as $S_{1}^{3}$. Finally $P_{G}$ is isomorphic to a bundle which is trivial outside $G \times D_{1}^{4}$ and transition function $g_{1,1}=\sigma_{l} \rho \sigma_{k}$.

Let us consider the case that the bundle $P_{0}$ over $Z_{0}$ is an arbitrary $S O(3)$ bundle but that $P_{1}$ can be lifted to an $S U(2)$-bundle. This process can be performed over $Z_{1}$ without much change. In fact, we only need to use the local trivialization of $P_{1}$. Finally $P_{G}$ can be constructed in the following way: 
We have a bundle $P_{0}$ over $Z_{0}$ which is trivialized over $Z_{0} \cap Z_{1}$. One can extend trivially over $G \times\left(Z_{1}-D_{1}^{4}\right)$. So we can view $P_{0}$ as a $S O(3)$-bundle over $G \times\left(X-D_{1}^{4}\right)$. A fixed trivialization over $G \times D_{1}^{4}, P_{G}$ can be obtained by the transition function $g_{1,1}: G \times S^{3} \rightarrow S O(3)$ where $g_{1,1}=\sigma_{l} \rho=\sigma_{l} \rho \sigma_{-l} \sigma_{l}$. So $P_{G}$ can also be obtained by first performing a transition function $\sigma_{l}$ and then $\sigma_{l} \rho \sigma_{-l}$. The first step only modifies $P_{0}$ to a bundle $P^{\prime}$, which is obtained by gluing a $S U(2)$-bundle with Chern class $l$ to $P_{0}$. In particular, $P^{\prime}$ has the same $w_{2}$ as $P_{0}$, and $p_{1}\left(P^{\prime}\right)=p_{1}\left(P_{0}\right)+4 l$.

Now we focus our attention on the last bundle gluing process, given by the map $\Phi_{l}: G \times S^{3} \rightarrow S O(3)$ where $\Phi_{l}(\rho, x)=\sigma_{l}(x) \rho \sigma_{-l}(x)$. Let $G=S^{1} \subset$ $S O$ (3) represent the generator of $S O(3)$. The case in which we are interested is the bundle $P_{S^{1}} \rightarrow S^{1} \times X$. Clearly $\sigma_{l}=\sigma \circ J^{l}$ where $J^{l}: S^{3} \rightarrow S^{3}$ is a degree $l$ map and $\sigma$ is the projection map $S U(2)$ to $S O(3)$. Since $\rho=1$ implies $\Phi_{l}=1, \Phi_{l}$ factors through a map:

$$
\widetilde{\Phi}_{l}: S^{1} \times S^{3} / 1 \times S^{3} \rightarrow S O(3) .
$$

Because of the homotopy equivalence $S^{1} \times S^{3} / 1 \times S^{3} \simeq \Sigma\left(S^{3}\right) \vee S^{1}$, we can assume that $\widetilde{\Phi}_{l}: \Sigma\left(S^{3}\right) \rightarrow S O(3)$, such that the map sends the two end points of the suspension to the same point. Furthermore, $\widetilde{\Phi}_{l}=\widetilde{\Phi} \circ \Sigma\left(J^{l}\right)$, where

$$
\Sigma\left(J^{l}\right): \Sigma\left(S^{3}\right) \rightarrow \Sigma\left(S^{3}\right)
$$

is the suspension map (degree $l$ self-map of $S^{4}$ ), and $\widetilde{\Phi}=\widetilde{\Phi}_{1}$. We claim that $\widetilde{\Phi}$ is the generator of $\pi_{4}(S O(3))=Z_{2}$. Given this claim we get that $\widetilde{\Phi}_{l}$ represents $l$-times the generator of $\pi_{4}(S O(3))$. From the description of $\pi_{1}\left(\mathscr{B}^{*}\right)$ from the last section we see that in fact this is also the generator of $\pi_{1}\left(\mathscr{B}^{*}\right)$.

It remains to prove the claim. First observe that since the map $\widetilde{\Phi}$ is given by $\widetilde{\Phi}(t, x)=x t x^{-1}$ where $t$ is the suspension coordinate, it factors as

$$
S^{4}=\Sigma\left(S^{3}\right) \rightarrow S^{3} \rightarrow S O(3)
$$

where the first map is easily seen to be the suspension of the Hopf map and the second map is the projection $\sigma$. Hence the claim follows.

\section{UNIVERSAL CONSTRAINTS}

Now we are in the position to prove the main theorem by using the cobordism construction of [R]. Let $P \rightarrow X$ be a $S O(3)$-principal bundle. By Chern-Weil theory, the necessary condition for $P$ to admit an ASD connection is that $p_{1} \leq 0$. Furthermore, by the Atiyah-Singer theorem [DK]

$$
\operatorname{dim}\left(\mathscr{M}_{w_{2}, p_{1}}\right)=-2 p_{1}-3\left(1+b_{2}^{+}\right) \text {. }
$$

So when $b_{2}^{+}(X)$ is odd $\operatorname{dim} \mathscr{M}_{w_{2}, p_{1}}=2 b$ for an integer $b$. Recall also that we have the Donaldsons $\mu$-map:

$$
\mu: H_{2}(X ; Z) \rightarrow H^{2}\left(\mathscr{B}^{*} ; Q\right)
$$

given by the homology slant product $\mu(\alpha)=-\frac{1}{4} p_{1}\left(\xi_{P}\right) / \alpha$ with the first Pontryagin class $p_{1}\left(\xi_{P}\right)$ of the universal $S O(3)$-bundle constructed in $\S 2$

$$
\xi_{P}=\mathscr{A}^{*} \times \widehat{\mathscr{G}} P \rightarrow \mathscr{B}^{*} \times X .
$$

By [K] this bundle lifts to an $U(2)$-bundle $\Xi_{P} \rightarrow \mathscr{B}^{*} \times X$ when $X$ is simply connected and $w_{2}(P) \neq 0$. Now for the rest of this section assume $X$ is simply connected and $w_{2}(P) \neq 0$. 
Lemma 3. Let $A_{2}(X)=\left\{\alpha \in H_{2}(X ; Z) \mid w_{2}(\alpha)=0\right\}$; then $\mu$ maps this set to the integral classes

$$
\mu: A_{2}(X) \rightarrow H^{2}\left(\mathscr{B}^{*} ; Z\right)
$$

Proof. If $w_{2}(\alpha)=0$, we can choose a lifting $\Xi_{P} \rightarrow \mathscr{B}^{*} \times X$ with $c_{1}(1 \times \alpha)=0$ Then $-p_{1}\left(\xi_{P}\right)=4 c_{2}\left(\Xi_{p}\right)-c_{1}^{2}\left(\Xi_{P}\right)$. By decomposing $c_{1}\left(\Xi_{P}\right)=c_{1}\left(\mathscr{B}^{*}\right) \times 1+1 \times$ $c_{1}(P)$ we get $c_{1}^{2}\left(\Xi_{P}\right)=c_{1}^{2}\left(\mathscr{B}^{*}\right) \times 1+1 \times c_{1}^{2}(P)+2 c_{1}\left(\mathscr{B}^{*}\right) \times c_{1}(P)$; hence,

$$
-p_{1}\left(\xi_{P}\right) / \alpha=4 c_{2}\left(\Xi_{P}\right) / \alpha-2 c_{1}\left(\mathscr{B}^{*}\right) \times c_{1}(P)(\alpha)=4 c_{2}\left(\Xi_{P}\right) / \alpha .
$$

Hence $\mu(\alpha)=-\frac{1}{4} p_{1}\left(\xi_{P}\right) / \alpha=c_{2}\left(\Xi_{P}\right) / \alpha$ is an integral class.

Proof (of the main theorem). Consider $\mathscr{M}_{w_{2}, p_{1}-4}$. Note that $\alpha \cdot \alpha=0 \bmod (2)$ iff $w_{2}(\alpha)=0$. For any $\alpha_{1}, \ldots, \alpha_{b+2} \in H_{2}(X, Z)$ with $w_{2}\left(\alpha_{i}\right)=0, \mu\left(\alpha_{i}\right)$ are integral classes. Choose the surfaces $\Sigma_{1}, \ldots, \Sigma_{b+2}$ in general position representing $\alpha_{1}, \ldots, \alpha_{b+2}$. We can define divisors $V_{\Sigma_{i}}$ that are transverse to each other and to $\mathscr{M}_{w_{2}, p_{1}}$ for $\dot{p} \geq p_{1}-4$. Recall the number of points of $V_{\Sigma_{1}} \cap \cdots \cap V_{\Sigma_{b}} \cap \mathscr{M}_{w_{2}, p_{1}}$, counted by orientation, is defined to be the Donaldson invariant $\Phi_{w_{2}, p_{1}}\left(\alpha_{1}, \ldots, \alpha_{b}\right)$. Let

$$
N=V_{\Sigma_{1}} \cap \cdots \cap V_{\Sigma_{b+2}} \cap \mathscr{M}_{w_{2}, p_{1}-4} .
$$

Then $\operatorname{dim} N=4$. $N$ is not compact. The ends of $N$ compactify to give cones on $S O(3)$. Each end corresponds a pair $([A], x)$ where

$$
[A] \in V_{\Sigma_{1}} \cap \cdots \cap \widehat{V}_{\Sigma_{i}} \cap \cdots \cap \widehat{V}_{\Sigma_{j}} \cap \cdots \cap V_{\Sigma_{b}} \cap \mathscr{M}_{w_{2}, p_{1}} \quad \text { and } \quad x \in \Sigma_{i} \cap \Sigma_{j} .
$$

The number of such pairs are measured by $q \circ \bar{\Phi}_{w_{2}, p_{1}}\left(\alpha_{1}, \ldots, \alpha_{b+2}\right)$. Cutting the cone, we get a compact manifolds with boundary $\bar{N} \subset N$. Furthermore, each component of its boundary is the gluing parameter $S O(3)$. By our assumption of $p_{1}-4=w_{2}^{2} \bmod 8$ and $w_{2}(P)=w_{2}(X), u_{1} \in H^{1}\left(\mathscr{B}^{*} ; Z_{2}\right)$ exists and $\left.u_{1}\right|_{S O(3)}$ gives the generator of $H^{1}\left(S O(3) ; Z_{2}\right)$ by the previous section. Hence,

$$
\begin{aligned}
0 & =u_{1}^{3}(\partial \bar{N})=q \circ \Phi_{w_{2}, p_{1}}\left(\alpha_{1}, \ldots, \alpha_{b+2}\right) u_{1}^{3}(S O(3)) \\
& =q \circ \Phi_{w_{2}, p_{1}}\left(\alpha_{1}, \ldots, \alpha_{b+2}\right)(\bmod 2) .
\end{aligned}
$$

(See Figure 5.)

Proof (of Corollary). Let $\Phi_{0}$ be a zero-degree Donaldson invariant. By theorem, $q \circ \Phi_{0}\left(\alpha_{1}, \alpha_{2}\right)=0 \bmod 2$ if $w_{2}\left(\alpha_{i}\right)=0$. But $q \circ \Phi_{0}\left(\alpha_{1}, \alpha_{2}\right)=$ $q\left(\alpha_{1}, \alpha_{2}\right) \Phi_{0}$. It is enough to show that there are classes $\alpha_{1}, \alpha_{2}$ such that $w_{2}\left(\alpha_{1}\right)=w_{2}\left(\alpha_{2}\right)=0$ and $q\left(\alpha_{1}, \alpha_{2}\right)=1$. When the manifold is odd, its intersection form is equivalent to $\bigoplus_{k}(1) \bigoplus_{n-k}(-1)$. Let $x_{1}, \ldots, x_{n}$ be the generators. Since $X$ is simply connected, $w_{2}$ can also be lifted as the integer

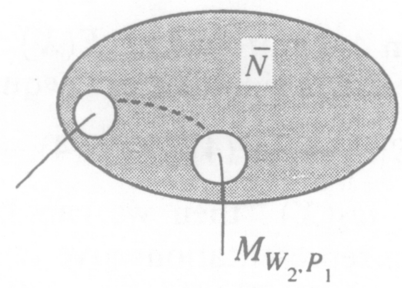

FIGURE 5 
class. In fact, one lift is $x_{1}+x_{2}+\cdots+x_{n}$. If $b_{2}=n \geq 3$, let $\alpha_{1}=x_{1}+x_{2}$ and $\alpha_{2}=x_{1}+x_{3}$. It is easy to check that $w_{2}\left(\alpha_{1}\right)=w_{2}\left(\alpha_{2}\right)=0$ but $q\left(\alpha_{1}, \alpha_{2}\right)=1$.

\section{INDEX THEORY CONSTRUCTION OF $u_{1}$}

Let $S O(3) \rightarrow \bar{P} \rightarrow X$ be a principal $S O(3)$-bundle. It is classified by the first Pontryagin class $p_{1}$ and second Steifel-Whitney class $w_{2}$ subject to the relation $w_{2}^{2}=p_{1}(\bmod 4)$.

There is an exact sequence

$$
0 \rightarrow S^{1} \rightarrow U(2) \rightarrow S O(3) \rightarrow 0
$$

where $S^{1}$ represents the diagonal matrices. When $X$ is simply connected, $w_{2}$ always has an integral lift. Any integral lift $c$ defines a lifting of the $S O(3)$ bundle to the $U(2)$-bundle $E \rightarrow X$ with $c_{1}=c$. The characteristic classes are related by

$$
\begin{gathered}
c_{1}(E)=c\left(=w_{2}(P) \bmod 2\right), \\
c_{1}^{2}(E)-4 c_{2}(E)=p_{1}(P) .
\end{gathered}
$$

When we restrict to the subgroup $S U(2) \subset U(2)$, we have

$$
0 \rightarrow Z_{2} \rightarrow S U(2) \rightarrow S O(3) \rightarrow 0 .
$$

This corresponds to a choice of the spin structure on $P$. In this case, $w_{2}(P)=0$ and $p_{1}=-4 c_{2}(E)$.

Under this lift, we can then interpret connections on $P$ as connections on $E$ which induce the fixed connection on the determinant; moreover, irreducible connections correspond to irreducible ones and ASD connections to ASD ones. Let $\mathscr{S} A_{*}(E)$ be the space of (irreducible) connections on $E$ inducing the fixed connection on the determinant. Let $\mathscr{S} G(E)$ be the group of gauge transformations of $E$ preserving the determinant. Then

$$
\mathscr{B}^{*}(P) \cong S \mathscr{B}^{*}(E)=\frac{\mathscr{S} A^{*}(E)}{\mathscr{S} G(E)} .
$$

For any $g \in \mathscr{S} G(E), \operatorname{det}(g)$ is a gauge transformation on $\operatorname{det}(E)$. Since $\operatorname{det}(g)$ preserves a fixed connection on $\operatorname{det}(E), \operatorname{det}(g)$ must be constant. Note that any element $x \in U(2)$ can be written as $x=x_{0} v$ for $x_{0} \in S U(2)$ and $v \in S^{1}$. Therefore, we can split $g=g_{0} v$ with $\operatorname{det}\left(g_{0}\right)=1, v \in S^{1}$ as a constant gauge transformation. It is easy to see that center $C(U(2))=S^{1}$. Hence $v \in \operatorname{stab}\left(\mathscr{S} A^{*}(E)\right)$. Then if $S_{0} \mathscr{G}(E)=\{g \in \mathscr{S} G(E), \operatorname{det}(g)=1\}$,

$$
\mathscr{B}^{*}(P)=\mathscr{S O B}^{*}(E)=\frac{\mathscr{S} A^{*}(E)}{S_{0} \mathscr{G}(E)} .
$$

Now let $X$ be a Riemannian 4-manifold and $F(X)$ be the frame bundle with structure group $S O(4)$. There is a similar exact sequence

$$
0 \rightarrow S^{1} \rightarrow \operatorname{Spin}^{c}(4) \rightarrow S O(4) \rightarrow 0 .
$$

If $f$ is an integral lift of $w_{2}(X)$, then we can lift $F(X)$ to a $\operatorname{Spin}^{c}(4)$ bundle. The irreducible Spin representations give two $U(2)$-bundles $V^{ \pm}$with first Chern class $f$. Consider $V^{ \pm} \otimes E$. When $w_{2}(P)=w_{2}(X)$, we can choose $c$ and $f$ such that $f=-c$. We claim that $V^{ \pm} \otimes E$ have real structures. Note 
that $V^{ \pm} \otimes E$ are the associated vector bundles of a $U(2) \times U(2)$ principal bundle through the representation on $C^{2} \otimes C^{2}$.

There is a subgroup in $U(2) \times U(2)$ as follows:

$$
G=\{(A, B) \in U(2) \times U(2) ; \operatorname{det}(A) \operatorname{det}(B)=1\} .
$$

$G$ contains a circle subgroup $S^{1}=\left\{\left(e^{i \theta}, e^{-i \theta}\right)\right\}$. Furthermore, $G / S^{1} \cong$ $S O(4)$. When $f=-c$, the structure groups of $V^{ \pm} \otimes E$ reduce to $G$ or $V^{ \pm} \otimes E$ are associated $G$-bundles by representations on $C^{2} \otimes C^{2}$. It is obvious that $S^{1}$ acts trivially on $C^{2} \otimes C^{2}$. So representations factor through $S O(4)$ and are the complexifications of the standard real representation. In other words, $V^{ \pm} \otimes E$ can be viewed as the complexification of real bundles.

Another way to view this construction is the following: First of all, identify $C^{2}$ with quaternion $H$ by $(a, b) \leftrightarrow a+J b$ with $J^{2}=-1$. Then we can also identify the corresponding $U(2)$ action. It is easy to check

$$
\begin{gathered}
\left(\begin{array}{cc}
\alpha & -\bar{\beta} \\
\beta & \bar{\alpha}
\end{array}\right) \in S U(2) \leftrightarrow \text { multiply by }(\alpha+J \beta) \text { from left } \\
e^{i \theta} I \leftrightarrow \text { multiply by } e^{i \theta} \text { from right. }
\end{gathered}
$$

Note that the multiplication of $J$ from right commutes with $S U(2)$ but not scalar multiplication. Now consider $C^{2} \otimes C^{2} \cong H \otimes H$. There is a conjugation map by multiplication of $J \otimes J$ from right. It obviously commutes with $S U(2) \times S U(2)$. Note that every element of $G$ can be expressed as the product of an element of $S U(2) \times S U(2)$ and $\left(e^{i \theta}, e^{-i \theta}\right)$. As we saw before, the second element acts trivially on $C^{2} \otimes C^{2}$. So $G$ commutes with $J \otimes J$. Note that $(J \otimes J)^{2}=1$. Then we decompose $C^{2} \otimes C^{2}$ as \pm -eigenspace and $i$ sends one to another. Furthermore, this decomposition commutes with $G$. This shows that the bundle itself is the complexification of a real bundle induced by +1 -eigenspace. It is straightforward to check that the coupled Dirac operator $D_{E}^{f}$ is compatible with the real structure. For the details we refer the reader to Chapter 3 of [DK]. Therefore, the kernel and cokernel of the operator are naturally real vector spaces.

Based gauge group $\mathscr{S} G_{0}=\left\{g \in \mathscr{S} G,\left.g\right|_{x_{0}}=1\right\}$ acts freely on the spaces of irreducible connections $\mathscr{S} A^{*}$ and commutes with $D_{E}^{f}$. Thus the index of the family of Dirac operators gives a virtual bundle

$$
\operatorname{Ind}\left(D_{E}^{f}\right) \in K O\left(\frac{\mathscr{S} A^{*}}{\mathscr{S} G_{0}}\right) .
$$

We want to push down bundle to the $\mathscr{S} B^{*}$. The obstruction to doing this is that -1 acts trivially on $\mathscr{S} A^{*}$ but possibly nontrivially on $\operatorname{Ind}\left(D_{E}^{f}\right)$. An index theorem calculation shows that -1 acts on the $\operatorname{det}\left(\operatorname{Ind}\left(D_{E}^{f}\right)\right)$ as $(-1)^{\operatorname{ind}\left(D_{E}^{f}\right)}$. Thus when index $\left(D_{E}^{f}\right)=0(\bmod 2), \operatorname{det} \operatorname{Ind}\left(D_{E}^{f}\right)$ descends to $\mathscr{S} B^{*}$. By the Atiyah-Singer Index Theorem,

$$
\operatorname{ind}\left(D_{E}^{f}\right)=2 \operatorname{ind}\left(D^{f}\right)-c_{2}(E)+\frac{1}{2}\left(f c_{1}(E)+c_{1}^{2}(E)\right) .
$$

By our choice, $f=-c_{1}(E)$. Hence $\operatorname{ind}\left(D_{E}^{f}\right)=c_{2}(E)(\bmod 2)$. Furthermore, $c_{2}(E)=0(\bmod 2)$ is equivalent to $p_{1}(P)=w_{2}^{2}(\bmod 8)$. Thus 
Definition. If $w_{2}(P)=w_{2}(X)$ and $p_{1}(P)=w_{2}^{2}(\bmod 8)$, we can choose liftings $c, f$ such that $f=-c$ and define

$$
u_{1}=w_{1}\left(\operatorname{Ind}\left(D_{E}^{f}\right)\right) \in H^{1}\left(\mathscr{S} B^{*}, Z_{2}\right) \text {. }
$$

Next we want to evaluate this class on the gluing parameter. Recall the gluing construction in $\S 3$. If we glue two $U(2)$ connections, then for each $\rho \in U(2)$ we can construct a $U(2)$-bundle $P_{\rho}$. Obviously we can also glue the induced det bundle by $\operatorname{det} \rho$. If we want the glued connection to have the same determinant, we have to fix det $\rho$, i.e., choose a smaller gluing parameter $S U(2)$. The rest of the argument is the same as Donaldson's excision argument to reduce the calculation to the one for the standard instanton on the 4-sphere. Finally we can prove that over the gluing parameter of a connection $A$ with the standard instanton of $S^{4}, \operatorname{Ind}\left(D_{E}^{f}\right)$ is $m+\eta$ where $m=\operatorname{ind}\left(D_{A}^{f}\right)$ and $\eta$ is the Hopf line bundle over $S O(3)$. So $\operatorname{det}\left(\operatorname{Ind}_{E}^{f}\right)=\eta$ and $\left.u_{1}\right|_{S O(3)} \neq 0$. In fact, $u_{1}^{3}(S O(3))=1$.

\section{REFERENCES}

[A] S. Akbulut, On universal relations in gauge theory (to appear).

[AJ] M. F. Atiyah and J. D. S. Jones, Topological aspects of Yang-Mills theory, Comm. Math. Phys. 61 (1978), 97-118.

[DW] A. Dold and H. Whitney, Classification of oriented sphere bundles over a 4-complex, Ann. of Math. (2) 69 (1959), 667-677.

[D1] S. K. Donaldson, Connections, cohomology and the intersection forms of 4-manifolds, $\mathbf{J}$. Differential Geom. 24 (1986), 275-341.

[D2] _- Polynomial invariants for smooth 4-manifolds, Topology 29 (1990), 257-315.

[D3] _ The orientation of Yang-Mills moduli spaces and 4-manifold topology, J. Differential Geom. 26 (1987), 387-428.

[DK] S. K. Donaldson and P. Kronheimer, The geometry of 4-manifolds, Oxford Math. Monographs, 1990.

[FS] R. Fintushel and R. Stern, SO(3)-connections and the topology of 4-manifolds, J. Differential Geom. 20 (1984), 523-539.

[K] D. Kotschick, $S O(3)$-invariants for 4-manifolds with $b_{2}^{+}=1$ (to appear).

[M] J. Morgan, Lecture notes.

[R1] Y. Ruan, A universal constraint on Donaldson invariants, J. Differential Geom. (to appear).

[R2] - Reducible connection on non-simply connected 4-manifolds (to appear).

[T] C. Taubs, Self-dual connections on manifolds with indefinite intersection matrix, J. Differential Geom. 19 (1984), 517-560.

Department of Mathematics, Michigan State University, East Lansing, Michigan 48824-0001

E-mail address: akbulut@math.msu.edu

Department of Mathematics, California Institute of Technology, Pasadena, CaliFORNIA 91125

Department of Mathematics, University of Utah, Salt Lake City, Utah 84112 